\title{
Influência das afecções de casco, cetose, lipidose hepática e outras sobre o desempenho reprodutivo de vacas leiteiras de elevada produção
}

\author{
Lameness, ketosis, hepatic lipidosis and other disorders \\ on the reproductive performance in dairy cows
}

Gilmar Godinho da Silva ${ }^{[a]}$, Luiz Ernandes Kozicki[ ${ }^{[b]}$,Alceu Miguel Grebogi[ib] Marcio Saporski Segui ${ }^{[b]}$, Romildo Romualdo Weiss ${ }^{[c]}$, Hugo Lopes Borges ${ }^{[a]}$, Francisco Gaievski[d]

[a] Acadêmicos do curso de Medicina Veterinária da Pontifícia Universidade Católica do Paraná (PUCPR), Curitiba, PR Brasil, e-mails: gilmarg450@hotmail.com; hugo-borges@hotmail.com

[b] Mestres em Ciência Animal, docentes da Pontifícia Universidade Católica do Paraná (PUCPR), Curitiba, PR - Brasil, e-mails: kozicki.l@pucpr.br; alceu.grebogi@pucpr.br; msegui@uol.com.br

[c] Doutor em Tierarztliche Hochschule pela Gottfried Wilhelm Leibniz Universität, Hannover, Alemanha, docente da Pontifícia Universidade Católica do Paraná (PUCPR), Curitiba, PR - Brasil, e-mail: rrweiss@bol.com.br

[d] Mestre em Ciência Animal pela Pontifícia Universidade Católica do Paraná (PUCPR), Curitiba, PR - Brasil, e-mail: francisco.embrasemen@hotmail.com

\section{Resumo}

O presente estudo objetivou verificar a influência da cetose, das afecções de casco, da lipidose hepática, do deslocamento de abomaso, do cisto folicular ovariano e de endometrite sobre o desempenho reprodutivo de vacas leiteiras de elevada produção e sobre a taxa de descarte animal. Foram levantados dados individuais relativos a 2008, 2009 e 2010, de vacas da raça Holandesa Preta e Branca com produção média de leite de 10.750 litros/305 dias/vaca. Dois grupos (G) de animais foram constituídos: o G1 (enfermos, $\mathrm{n}=38$ ) constou de animais acometidos por uma ou mais afecções, como cetose clínica, afecções de casco (laminite), lipidose hepática, deslocamento de abomaso, cisto folicular ovariano ou endometrite; e o G2, formado por animais sadios $(\mathrm{n}=25)$. No tratamento estatístico dos dados foram utilizados os testes do Qui-Quadrado e o de Mann-Whitney. Os resultados demonstraram a incidência de, respectivamente, 68,4\%; 23,6\%; 15,7\%; $13,1 \% ; 15,7 \%$ e $7,8 \%$ de cetose, afecções de casco, lipidose hepática, deslocamento de abomaso, cisto folicular ovariano e endometrite. Houve diferença de incidência entre a cetose e as outras afecções citadas ( $\mathrm{p}<0,05)$. Ocorreu, nesta ordem, maior número de serviços/prenhez, maior taxa de descarte de animais, maior intervalo parto/concepção para as variáveis cetose, afecções de casco, lipidose hepática e cisto folicular ovariano $(p<0,05)$, deslocamento de abomaso e endometrite $(p>0,05)$ nos animais enfermos do que nos sadios. A taxa de prenhez entre os grupos foi de 78,9\% e 92,0\%, respectivamente, para os animais enfermos e sadios $(p>0,05)$. Concluiu-se que as afecções pesquisadas no pós-parto reduziram a performance reprodutiva dos animais enfermos em relação aos sadios, destacando-se os cistos foliculares, as afecções de casco, a cetose e a lipidose hepática sobre o intervalo parto/concepção $(p<0,05)$, além do deslocamento 
do abomaso e das endometrites ( $p>0,05)$; e que os animais enfermos tiveram maior número de serviços/ prenhez, maior taxa de descarte $(p<0,05)$ e menor taxa de prenhez $(p>0,05)$.

Palavras-chave: Cetose. Laminite. Cisto folicular. Vacas leiteiras.

\section{Abstract}

The objective of the present study was to verify the influence of ketosis, laminess, hepatic lipidosis, displaced abomasum, ovarian follicular cysts and endometritis on the reproductive performance and culling rates of highly productive dairy cows. Data were collected from individual records (2008-2010) of Holstein Friesian Black and White cows with average milk production of 10,750 liters/305 days/cow. The animals were divided in two groups $(G): G 1(n=38)$ was consisted of animals with ketosis, laminess, hepatic lipidosis, displaced abomasum, endometritis and ovarian follicular cysts; and G2 was composed by healthy animals ( $n=25)$. Chi-square and MannWhitney tests were used for statistical analysis. The results showed an illness incidence of 68.4\% for ketosis; 23.6\% for laminess; $15.7 \%$ for hepatic lipidosis; 13.1\% for displaced abomasums; 15.7\% for ovarian follicular cysts; and 7.8\% for endometritis. There was a significantly greater incidence of ketosis in comparison to the other diseases. Moreover, sick animals had a greater number of artificial insemination (AI)/pregnancy, longer interval of parturition-to-conception and higher rate of discharge in comparison to healthy individuals. The final pregnancy rate was $78.9 \%$ and $92.0 \%$ ( $p>0.05$ ) for sick and healthy animals, respectively. Therefore, it can be concluded that the disorders presented in postpartum dairy cows contributed significantly to increase the number of AI/pregnancy, increase the open days between calving and conception, and increase the rate of animal discharge.

Keywords: Ketosis. Laminitis. Follicular cysts. Milk cows.

\section{Introdução}

As afecções digitais dos bovinos acarretam impacto econômico negativo sobre a rentabilidade da pecuária, reduzindo a produtividade e aumentando os custos dos tratamentos, além do prematuro descarte de animais de elevado valor zootécnico (CORBELLINI, 1994). Ramos et al. (2001), avaliando os parâmetros reprodutivos em vacas leiteiras, encontraram $25 \%$ de taxa de descarte de animais com enfermidades digitais. Silva (2002) confirmou a importância econômica das doenças de casco destacando os seus efeitos sobre a produção, a menor eficiência reprodutiva dos touros, o aumento no período de serviço e os altos custos dos tratamentos. As afecções dos cascos podem constituir-se em importante fator limitante na manutenção de bons níveis de fertilidade, muito embora a associação dessas enfermidades com a redução dos níveis de fertilidade seja passível de questionamentos.

No Brasil, as doenças digitais dos bovinos somente perdem em prejuízos econômicos para as mastites e as patologias da reprodução, afetando de $11 \%$ a $25 \%$ das vacas leiteiras (DIAS, 1996). Estudos con- duzidos por Orenisk (2005), na Eslovênia em vacas leiteiras Friesian, evidenciaram 15,1\% de enfermidades digitais, sendo que diversas vacas portavam problemas reprodutivos associados a essas afecções. A retenção de anexos fetais em bovinos pode acarretar as endometrites puerperais (GRUNERT BERCHTOLD, 1995). Nilsson (1963) relatou que a histamina, produzida em úteros portadores de retenção de anexos fetais e de endometrites, poderia desencadear a laminite. Para Boosman et al. (1991), as endotoxinas produzidas nesses casos estão relacionadas com a etiopatogenia das enfermidades podais. Allenstein (1981), Edwards (1982) e Liversey e Fleming (1984) sinalizaram que a histamina e as endotoxinas decorrentes das doenças sistêmicas no puerpério, como a acetonemia, a mastite, a metrite e a acidose ruminal, são responsáveis por danos ao complexo laminar podal. Esses pesquisadores chamaram a atenção para o fato de a laminite aguda ter sido observada em animais portadores de doenças com a presença de endotoxinas.

Em estudos efetuados por Vermunt e Greenough (1994), verificou-se a ocorrência de laminites em bovinos submetidos a elevadas doses de 
endotoxinas administradas via artérias digitais. Silva (2002) acrescenta que o excesso de grãos, milho e rações substancialmente proteicas, além das enfermidades da glândula mamária e do útero, são fatores de risco direcionados à laminite. Peeler et al. (1994) constataram significativa associação entre as afecções de cascos e de mastite. Os autores sinalizaram que fatores relacionados ao manejo poderiam predispor o animal a ambas as condições. Eles ainda ressaltam que as distocias fetais constituíam fatores significativos para o surgimento de mastites e doenças podais, deduzindo que os traumatismos intrapartais aumentavam a susceptibilidade às doenças comuns no puerpério.

No final da gestação e no início da lactação, existe maior risco de doenças metabólicas em vacas leiteiras especializadas, quando comparadas com as outras fases do ciclo de lactação (CONTI et al., 2002). Segundo Duffield et al. (1999), a principal predisposição a tais distúrbios acontece em virtude da extrema demanda de energia com maior mobilização de gordura corpórea e a redução na capacidade de ingestão de matéria seca, afetando a produção leiteira e a reprodução. A cetose e a lipidose hepática são doenças inter-relacionadas, associadas ao balanço energético negativo e à carência de carboidratos precursores de glicose, típicas do periparto de vacas de alta produção leiteira. Nesse período também ocorre o período de reprodução, fato importante a se considerar, uma vez que o aumento das demandas metabólicas reduz a fertilidade das vacas, reduzindo a meta de se obter um bezerro por ano (WITTWER, 2000).

A cetose incide sobre bovinos leiteiros de elevada produção. Existe uma redução da concentração de glicose e um aumento significativo dos corpos cetônicos sanguíneos. Ao iniciar a lactação, vacas leiteiras exigem significativa necessidade de energia, em função do aumento da produção. 0 balanço energético negativo (BEN) pode instalar-se pelo não acompanhamento das exigências nutricionais do animal neste período. Animais em boas condições de manejo e alimentação utilizam suas reservas corporais para a obtenção de energia. Porém, a quantidade de ácidos graxos utilizada principalmente a nível hepático atinge um limite. Ao se atingir o limite, os lipídios não serão utilizados para o fornecimento da energia, mas irão acumular-se nos hepatócitos como triglicerídeos, sendo alguns dos ácidos graxos convertidos em cetonas (GUARD, 1996; GOFF, 2006).
A lipidose hepática pode advir do desequilíbrio entre a captação hepática dos ácidos graxos e a sua utilização (AROEIRA, 1998). Define-se a lipidose hepática como a presença excessiva de lipídios no fígado, ocorrendo quando o índice de acumulação de triglicerídeos excede seus índices de degradação metabólica ou liberação como lipoproteínas (MACLACHLAN; CULLEN, 1998).

Existem outras enfermidades que podem afetar o desempenho reprodutivo (desordens gastroentéricas, respiratórias ou digestivas, como o deslocamento de abomaso), porém são escassos os estudos específicos relacionando essas doenças com o desempenho reprodutivo de vacas de elevada produção.

Kozicki (1982), Fantini Filho et al. (2003) e LopezGatius et al. (2008) estudaram vacas leiteiras de elevada produção leiteira com problemas ovarianos, detectando, respectivamente, 18,7\%, 50,8\% e $20,8 \%$ de animais com cisto folicular. Cisto folicular é definido, clinicamente, como uma vesícula com diâmetro de 20-25 mm (ou maior), que permanece em um dos ovários por um período de 7-10 dias ou mais. 0 folículo tem sua capacidade esteroidogênica alterada por processos degenerativos. Foi constatado que animais tratados com subdoses de progesterona desenvolvem um folículo dominante que persiste no ovário por período mais longo que o normal. A infertilidade resultante dos ciclos em que esses folículos chegam a ovular ocorre provavelmente em razão da má qualidade dos ovócitos liberados (CORREA et al., 2001).

Endometrite é a inflamação do útero que afeta o endométrio. Dentre os principais agentes causadores das endometrites estão os Streptococcus spp., Escherichia coli, Corynebacterium spp., Staphylococcus spp., Pseudomonas aeruginosa e a Klebsiella pneumoniae (KOZICKI et al., 1988; CORREA et al., 2001).

0 presente estudo objetivou verificar a influência da cetose, das afecções de casco, da lipidose hepática, do deslocamento de abomaso, do cisto folicular ovariano e de endometrite sobre o desempenho reprodutivo e sobre a taxa de descarte de vacas leiteiras de elevada produção.

\section{Materiais e métodos}

No presente estudo foram utilizados dados de vacas Holandesas Preta e Branca com produção 
média de 10.750 litros de leite em 305 dias, perfazendo uma produção média de 35,2 litros/dia/vaca. Foram utilizados dados de 63 animais entre os anos de 2008 e 2010. Foram analisadas fichas da incidência da cetose, das afecções de casco, da lipidose hepática, do deslocamento de abomaso, de cisto folicular ovariano e de endometrite sobre a performance reprodutiva e sobre a taxa de descarte animal. A análise dos dados possibilitou a formação de dois grupos (G) de animais: o G1 ( $\mathrm{n}=38$ animais enfermos), constituído por vacas que haviam sido acometidas por uma ou mais das afecções acima descritas no período pós-parto (até quatro semanas pós-parto); e o G2 ( $\mathrm{n}=25)$, composto por animais sadios. Além desses aspectos, de cada ficha individual foram verificados os dados de intervalo parto/ concepção, taxa de prenhez de cada grupo, número de serviços/prenhez e taxa de descarte animal. Os animais enfermos eram tratados a seu tempo, de acordo com os tratamentos convencionais. Para a reprodução foi adotada a inseminação artificial convencional após a observação visual do estro.

\section{Análise estatística}

Para as variáveis incidência das afecções nos animais, taxa de prenhez geral dos grupos e taxa de descarte, foi empregado o teste do Qui-Quadrado. Já para a verificação do intervalo (dias) entre o parto e a concepção e o número de serviços/prenhez, foi utilizado o Teste de Mann-Whitney. 0 nível de significância para todos os testes foi de $95 \%$ de confiabilidade.

\section{Resultados e discussão}

Os resultados obtidos estão representados nas tabelas a seguir.

Kozicki (1982), estudando vacas leiteiras em condições similares às do presente estudo, reporta várias afecções incidentes no puerpério. Os dados no presente estudo demonstraram diferenças $(\mathrm{p}<0,05)$ entre os animais com e sem afecções no puerpério. Ocorreram significativas alterações em alguns dos parâmetros reprodutivos avaliados, acarretando danos no sistema de produção, como acontece na bovinocultura leiteira de elevada produção. Praticamente todas as variáveis relacionadas à reprodução foram influenciadas pelas afecções pesquisadas.

A incidência da cetose despontou como a mais frequente das afecções $(68,4 \%)$ dentre as estudadas ( $p<0,05$ - Tabela 1). Ela geralmente ocorre entre os dias 8 e 60 do pós-parto, período em que o animal enfrenta um balanço energético negativo (BEN) (GONZÁLEZ; CAMPOS, 2003). No presente estudo, a cetose acarretou o aumento do intervalo entre o parto e a concepção (dias abertos) de 57,8 dias em relação ao grupo de vacas sadias (148,1 versus 90,3 dias), respectivamente, $\mathrm{p}<0,05$. Tais dados suplantam os obtidos por Anderson (1988), que relata 92 dias de intervalo parto/concepção nos animais afetados pela cetose. Miettinen (1991) e Anderson et al. (1991) observaram que essa afecção metabólica afetou negativamente o intervalo parto/primeira inseminação artificial, como o ocorrido neste estudo.

De acordo com Brizzi e Carrara (1993), os problemas de casco dos bovinos são considerados um dos grandes entraves à atividade leiteira, pois ocasionam a perda de peso dos animais, a redução da produção leiteira a níveis muito baixos e o isolamento do plantel, acarretando sérios riscos de vida, tornando-os candidatos à reposição. De acordo com Dias (1996), as afecções podais podem afetar de $11 \%$ a $25 \%$ das vacas de qualquer propriedade, corroborando com a cifra na presente pesquisa (24\%). As afecções podais no presente estudo afetaram o

Tabela 1 - Ocorrência de algumas enfermidades incidentes em vacas leiteiras da raça Holandesa Preta e Branca de elevada produção até o período de quatro semanas pós-parto $(n=38)$

\begin{tabular}{lcc}
\hline Afecção & $\begin{array}{c}\text { Animais } \\
\text { acometidos } \\
\text { (n) }\end{array}$ & $\begin{array}{c}\text { Percentual de } \\
\text { ocorrência } \\
\text { (\%) }\end{array}$ \\
\hline Cetose & $26 / 38$ & $68,4^{\mathrm{a}}$ \\
Afecções de casco & $9 / 38$ & $23,6^{\mathrm{b}}$ \\
Lipidose hepática & $6 / 38$ & $15,7^{\mathrm{b}}$ \\
Deslocamento de abomaso & $5 / 38$ & $13,1^{\mathrm{b}}$ \\
Cisto folicular ovariano & $6 / 38$ & $15,7^{\mathrm{b}}$ \\
Endometrite & $3 / 38$ & $7,8^{\mathrm{b}}$ \\
\hline
\end{tabular}

Fonte: Dados da pesquisa.

Legenda: Letras diferentes na mesma coluna $(p<0,05)$. 
Tabela 2 - Intervalo entre o parto e a concepção, número de serviços/prenhez, taxa de prenhez total e taxa de descarte total em vacas leiteiras com afecções e sem afecções até o período de quatro semanas pós-parto $(n=63)$

\begin{tabular}{lcccc}
\hline Variável & $\begin{array}{c}\text { Intervalo parto/ } \\
\text { concepção }(\mathbf{x} \pm \mathbf{s}) \text { (dias) }\end{array}$ & $\begin{array}{c}\text { N. de serviços/ } \\
\text { prenhez }(\mathbf{x} \pm \mathbf{s})\end{array}$ & $\begin{array}{c}\text { Taxa de prenhez total Taxa de descarte total } \\
\text { (\%) }\end{array}$ \\
\hline Cetose & $148,1 \pm 74,1^{\text {aA }}$ & & \\
Afecções de casco & $161,8 \pm 55,8^{\text {aA }}$ & & \\
Lipidose hepática & $138,5 \pm 83,7^{\text {aA }}$ & & \\
Cisto folicular ovariano & $169,0 \pm 60,6^{\text {aA }}$ & & \\
Deslocamento de abomaso & $117,2 \pm 85,9^{\text {bA }}$ & & \\
Endometrite & $118,5 \pm 34,5^{\text {bA }}$ & & $78,9^{\text {a }}$ \\
Vacas sadias & $90,3 \pm 29,6^{\mathrm{b}}$ & $2,54 \pm 1,7^{\mathrm{a}}$ & $92,0^{\mathrm{a}}$ \\
Animais com afecções (total) & & $1,36 \pm 0,6^{\mathrm{b}}$ & $8,0^{\mathrm{b}}$ \\
Animais sem afecções (total) & & & \\
\hline
\end{tabular}

Fonte: Dados da pesquisa.

Legenda: Letras minúsculas diferentes na mesma coluna $(p<0,05)$; letras maiúsculas iguais na mesma coluna $(p>0,05)$.

intervalo parto/concepção (161,8 versus 90,3 dias) $(\mathrm{p}<0,05)$, respectivamente nos animais enfermos e sadios, causando impacto sobre a reprodução.

Os distúrbios como a lipidose hepática e os cistos foliculares ( $\mathrm{p}<0,05)$, o deslocamento do abomaso e as endometrites igualmente afetaram o intervalo parto/concepção na presente pesquisa (Tabela 2), ao se comparar com o grupo de animais sadios. A lipidose hepática estaria de certo modo associada à cetose (GRUMMER, 1993). Formigoni et al. (1996) relacionam negativamente esses distúrbios referentes à baixa produção de GnRH e IGF-I, bem como a menor frequência de pulsos de hormônio luteinizante, acarretando prejuízos à performance reprodutiva. Segundo Fleming (1993), o deslocamento de abomaso associado à cetose são entidades nosológicas primárias comuns, conduzindo ao aparecimento da cetose e, assim, afetando diretamente a reprodução.

Cistos foliculares constituem uma das principais causas da infertilidade em vacas leiteiras (KOZICKI, 1982; MWAANGA; JANOWSKI, 2000; CORREA et al., 2001; FANTINI FILHO et al., 2003). Dados do presente estudo demonstraram que os cistos foliculares acarretaram o maior intervalo entre parto/ concepção (169,0 dias) dentre as afecções pesquisadas. Fantini Filho et al. (2003), em condições similares às do presente estudo, relataram o período de serviço de 137,1 dias em animais com cistos ovarianos tratados com hormonioterapia. Esses autores verificaram o longo período de tempo de 74,4 dias, em média, entre o tratamento e a prenhez, demonstrando a magnitude dos danos causados pelos cistos.

No confronto e no cômputo geral dos animais enfermos e sadios, verificou-se menor taxa de prenhez nos animais enfermos $(78,9$ versus $92,0-p>0,05)$, salientando-se ainda o aumento do número de serviços/prenhez (2,54 versus 1,36, respectivamente, para enfermos e sadios), assim como maior taxa de descarte de animais enfermos do que sadios, respectivamente $(15,7 \%$ versus $8,0 \% \mathrm{p}<0,05)$. Entre as afecções estudadas não houve diferença significante do intervalo parto/concepção, ressaltando-se, contudo, que os cistos foliculares determinaram maior prolongamento do intervalo, perfazendo 169,0 dias.

\section{Conclusão}

Concluiu-se que as afecções pesquisadas no pós-parto reduziram o desempenho reprodutivo dos animais enfermos, destacando-se os cistos foliculares, as afecções de casco, a cetose e a lipidose hepática sobre o intervalo parto/concepção $(p<0,05)$, além do deslocamento do abomaso e das endometrites ( $p>0,05)$; que os animais enfermos necessitaram de maior número de serviços/prenhez e tiveram menor taxa de prenhez ( $p>0,05)$, além de maior taxa de descarte $(p<0,05)$. 


\section{Referências}

ALLENSTEIN, L. C. Lameness of cattle. Canadian Veterinary Journal, v. 22, p. 65-67, 1981.

ANDERSON, L. et al. Effect of hyperketonaemia and feeding on fertility in dairy cows. Theriogenology, v. 36, n. 4, p. 521-536, 1991.

ANDERSON, L. et al. Subclinical ketosis in dairy cows. Veterinary Clinics of North America Food Animal Practice, v. 4, n. 2, p. 233, 1988.

AROEIRA, L. J. M. Cetose e infiltração gordurosa no fígado em vacas leiteiras. Juiz de Fora: EMBRAPA-CNPGL, 1998. Documentos, 65.

BOOSMAN, R. et al. Bovine Laminitis: clinical aspects, pathology and pathogeneses with reference to acute bovine laminitis. Veterinary Record, v. 125, p. 573-575, 1991.

BRIZZI, A.; CARRARA, F. E. Como evitar os maiores problemas de cascos. Revista Gado Holandês, v. 421, p. 3440, 1993.

CONTI, R. M. C. et al. Efeito da monensina na produção e composição do leite e parâmetros reprodutivos em vacas holandesas. In: REUNIÃO ANUAL DA SOCIEDADE BRASILEIRA DE ZOOTECNIA, 39., 2002, Recife. 2002. Anais... Recife: Nutrição de Ruminantes, CD-ROM. NUR-822.

CORBELLINI, C. N. Factores nutricionales de riesgo para las afecciones podales. In: JORNADA-TALLER PARA MÉDICOS VETERINÁRIOS, 1., 1994, Navarro. Anais... Navarro: JornadaTaller para Médicos Veterinários, 1994. p. 1-15.

CORREA, F. R. et al. Doenças de ruminantes e equinos. 2. ed. São Paulo: Varela, 2001. v. 2, p. 369-375.

DIAS, R. S. Tratamento de cascos se faz com informações e critérios. Revista Balde Branco, v. 385, n. 3, p. 26-29, 1996.

DUFFIELD, T. F. et al. Effect of prepartum administration of monensin in controlled-release capsule on milk production and milk components in early lactation. Journal of Dairy Science, v. 82, n. 2, p. 1254-1263, 1999.

EDWARDS, G. B. Acute and subacute laminitis in cattle. Veterinary Annuary, v. 106, n. 22, p. 99-106, 1982.

FANTINI FILHO, J. C. et al. Cistos ovarianos no puerpério de vacas com elevada produção leiteira. Revista Brasileira de Reprodução Animal, v. 27, n. 3, p. 561$563,2003$.
FLEMING, S. A. Cetose dos ruminantes (acetonemia). In: SMITH, B. P. Tratado de Medicina Interna de Grandes Animais. São Paulo: Editora Manole, 1993.v.2,p.1297-1304.

FORMIGONI, A. et al. Effect of propylene glycol supplementation around parturition on milk yield, reproduction performance and some hormonal and metabolic characteristics in dairy cows. Journal of Dairy Research, v. 63, n. 1, p. 11-24, 1996.

GOFF, J. Principais síndromes que acometem as vacas leiteiras no período periparto. In: WORLD BUIATRICS CONGRESS, 24., 2006, França. Proceedings... França: World Buiatrics Congress, 2006.

GONZÁLEZ, F. H. D.; CAMPOS, R. Indicadores metabólico-nutricionais do leite. In: I Simpósio de Patologia Clínica Veterinária da Região Sul do Brasil Porto Alegre, 1., 2003, Porto Alegre. Anais... Porto Alegre: Ed.UFRGS, 2003.p. 31-47.

GRUMMER, R. R. Etiology of lipid-related metabolic disorders in periparturient dairy cows. Journal of Dairy Science, v. 76, n. 12, p. 3882-3896, 1993.

GRUNERT, E.; BERCHTOLD, M. Fertilitätsstörungen beim weiblichen Rind. Berlin: Parey Verlag, 1995.

GUARD, C. L. Fresh cow problems are costly: culling hurts the most. Hoard's Dairyman, v. 141, p. 8, 1996.

KOZICKI, L. E. et al. Contribuição ao tratamento do anestro pós-puerperal em vacas leiteiras. Revista Setor de Ciencias Agrárias, v. 10, n. 1-2, p. 157-162,1988.

KOZICKI, L. E. Uber den postpartalen Zyklusverlauf bei Kuhen unter verschiedenen Haltungsbedingungen, dargestellt anhand von klinischen Erhebungen und von Progesteronbestimmungen in Milchproben mit Hilfe des Enzymimmuntests und Radioimmuntests. 1982. 186 f. Thesis (Doktor Veterinarmedizin) - Justus Liebig universitat Giessen, Germany, 1982.

LIVERSEY, C. T.; FLEMING, F. L. Nutritional influences on laminitis, sole ulcer and bruised sale in Friesian cows. Veterinary Record, v. 114, p. 510-512, 1984.

LOPEZ-GATIUS, F. et al. Factors affecting the response to the specific treatment of several forms of clinical anestrus in high producing dairy cows. Theriogenology, v. 69 , p. $1095-1103,2008$.

MACLACHLAN, N. J.; CULLEN, J. M. Fígado, sistema biliar e pâncreas exócrino. In: CARLTON, W. W.; McGAVIN, M. D. Patologia veterinária especial de Thompson. 2 . ed. Porto Alegre: ARTMED, 1998. p. 95-131. 
MIETTINEN, P. V. A. Correlation between energy balance and fertility in Finnish dairy cows. Acta Veterinaria Scandinavica, v. 32, n. 2, p. 189-196, 1991.

MWAANGA, E. S.; JANOWSKI, T. Anoestrus in dairy cows: causes, prevalence and clinical forms. Reproduction in Domestic Animals, v. 35, p. 193-200, 2000.

NILSSON, S. A. Clinical, morphological and experimental studies of laminitis in cattle. Acta Veterinaria Scandinavica, v. 4, p. 9-304. 1963. Supplement 1

ORENISK, A. Effect of health and reproductive desorders on milk yield and fertility in dairy cows. Bovine Practice, v. 29 , p. $33-44,2005$.

PEELER, E. J. et al. Inter-relationships of periparturiente diseases in dairy cows. Veterinary Record, v. 5, p. 129132,1994

RAMOS, L. S. et al. Avaliação de parâmetros reprodutivos em fêmeas bovinas de aptidão leiteira portadoras de pododermatite necrosante. Archives of Veterinary Science, v. 17, n. 2, p. 98-106, 2001.
SILVA, L. A. F. Mudanças trazem doenças de casco em Goiás. Revista DBO, n. 160, p. 262-156, 2002.

VERMUNT, J. J.; GRENOUGH, P. R. Predisposing factors of laminitis in cattle. British Veterinary Journal, v. 150, n. 2, p. $150-164,1994$.

WITTWER, F. Diagnóstico dos desequilíbrios metabólicos de energia em rebanhos bovinos. In: GONZÁLEZ, F. H. D.; BARCELLOS, J. O. J.; OSPINA, H. (Ed.). Perfil metabólico em ruminantes: seu uso em nutrição e doenças nutricionais. Porto Alegre: Gráfica da Universidade Federal do Rio Grande do Sul, 2000. p. 9-22.
Recebido: 26/09/2011

Received: 09/26/2011

Aprovado: 15/12/2011

Approved: 12/15/2011 